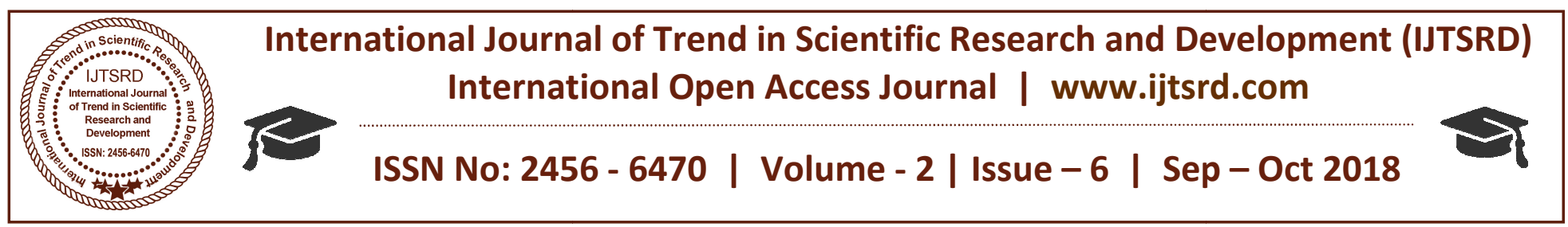

\title{
Intelligent Exercise System
}

\author{
Nitu Sharma \\ Student, Rajiv Gandhi College of Engineering Research and Technology, Chandrapur, Maharashtra, India
}

\begin{abstract}
Nowadays as the world is heading towards the smart cities and automation is available in each and every prospect. The automations are available in each and every field. The medical field is very important. In military and also for the person who is not physically fit. Those person required physiotherapy to monitor and making exercise to make fit. The cost of physical rehabilitation technique is too high for the long period. In this paper the kinet based technique for physical rehabilitation is design. The program is developed in MATLAB software. The algorithm is developed by using supervised learning model.
\end{abstract}

Keyword: Kinet, skeleton tracking, depth sensor.

\section{INTRODUCTION}

In preventive and rehabilitative social insurance, physical exercise is a great mediation. Be that as it may, a program may require in the scope of thousands of training redundancies, and numerous individuals don't hold fast to the program or play out their home activities mistakenly, making the activity incapable, or indeed, even perilous [1]. Exercise programs endorsed to address particular issues must be separately customized by a clinician because of the nearness of co-morbidities and extra disabilities. The present best in class for practice direction and observing is normally constrained to composed guidelines; work out recording logs, and basic reiteration tallying gadgets. Shockingly, this training has various issues:

$>$ The patient does not get any criticism on the nature of the recommended works out.

$>$ The clinician has no chance to get of knowing whether or not the patient has completed the endorsed works out effectively and with the required number of reiterations.

Amend adherence to supplemental home exercise is basic for sheltered, viable, and productive restoration mind [1]. The absence of rightness criticism on the inhome activities is in this way a genuine concern. The arrival of the Microsoft Kinect sensor, which is furnished with a profundity camera fit of estimating 3 dimensional (3D) places of the items in its see, and the comparing programming libraries that empower the accepting of skeletal joint positions in real time, have activated gigantic interests in utilizing the sensor to screen in-home restoration practices on the grounds that:

The Kinect sensor can be modified to record an activity session and give persistent input about remedy practice execution to the patient working out at home.

A Kinect-based framework could encourage legitimate execution of recovery practices at home, which would altogether limit treks to an active recuperation focus, which are expensive furthermore, badly arranged for patients. Moreover, such a framework could build understanding responsibility, enable the clinician to remedy any mistakes in practice execution, and permit program change or progression as required. For sure, a few achievability examines (counting our own) have shown that Kinect gives exact estimation to practices with great frontal view (e.g., [2]). In this paper, we depict the plan and usage of such a Kinect-based framework for recovery works out observing and direction. The framework exhibits the right method for completing an activity by means of a 3D symbol on one side of the screen in view of pre-recorded movement information. On the opposite side of the screen, another symbol is demonstrated that mirrors the genuine tolerant development. Besides, the framework executes a set of accuracy rules for each activity and evaluates the patient's development in real time. The appraisal results are joined in the patient symbol as visual advisers for help the quiet play out the activity effectively. The framework likewise records crucial 
information related to the quality and amount of activities such as right emphasess and nitty gritty movement information for real time criticism and post-investigation.

\section{BACKGROUND}

In this section, we provide more in-depth information regarding Microsoft Kinect. We also briefly introduce correctness rules for a few common rehabilitation exercises.

The Kinect sensor bar is an RGB-D camera introduces by Microsoft, which helps in developing gesture recognition systems by capturing depth information, and converting the captured information into $3 \mathrm{D}$ coordinates. The kinect sensor is shown in Figure 1.The kinect sensor consists of the following parts:-

\section{A. Kinect Hardware Specifications}

The Kinect for Xbox 360 has a RGB camera as the the color sensor, and a depth sensor comprised of an infrared (IR) light source emitter and an IR depth sensor. The later Kinect brings out new technologies with an IR illuminator and an IR Time-Of-Flight (TOF) depth sensor. Both Kinects and a detailed comparison of their specifications are shown in Fig. 1

\section{$[1,2,3,4]$.}

\begin{tabular}{|l|l|l|}
\hline Device & Kinect for Xbox 360 & Kinect for Xbox One \\
\hline Photo & & \\
\hline Color Camera & $640 \times 480 @ 30$ FPS & $1920 \times 1080$ @30 FPS \\
\hline Depth Camera & $320 \times 240$ & $512 \times 424$ \\
\hline $\begin{array}{l}\text { Min / Max Depth } \\
\text { Distance }\end{array}$ & $40 \mathrm{~cm} / 4.5 \mathrm{~m}$ & $50 \mathrm{~cm} / 8 \mathrm{~m}$ \\
\hline Horizontal Field Of View & 57 degrees & 70 degrees \\
\hline Vertical Field of View & 43 degrees & 60 degrees \\
\hline Tilt Motor & Yes & No \\
\hline $\begin{array}{l}\text { Number of Skeleton } \\
\text { Joints Identified }\end{array}$ & 20 & 25 \\
\hline $\begin{array}{l}\text { Number of Full } \\
\text { Skeletons Tracked }\end{array}$ & 2 & 6 \\
\hline USB Standard & 2.0 & 3.0 \\
\hline Minimum Latency (Lag) & $102 \mathrm{~ms}$ & $20 \mathrm{~ms}$ \\
\hline $\begin{array}{l}\text { Active IR (able to use in } \\
\text { dark/low light) }\end{array}$ & No & Yes \\
\hline
\end{tabular}

Fig.1. A comparison of specifications for Kinect versions

\section{B. Computer Vision Capabilities of Kinect}

Although it is categorized as a depth camera, the Kinect sensor is more than that. It has several advanced sensing hardware containing a color camera, a depth sensor, and a four-microphone array. These sensors ensure different opportunities at 3D motion capture, face and voice recognition areas [5]. While Kinect for Xbox 360 uses a structured light model to get a depth map of a scene, Kinect for Xbox One uses a faster and more accurate TOF sensor. After the arrival of Kinect, it did not take long that the computer vision community discovered the potential of Kinect that could extend far beyond gaming. Kinect costs much lower than traditional 3-D cameras (such as stereo cameras and time- of-flight (TOF) cameras) [6]. Not only indoor 3-D mapping, Kinect may be used for many other Computer Vision topics including object tracking and recognition, human activity analysis, and hand gesture recognition. A tree-structured classification in Fig.2 shows what kind of vision problems can be addressed or enhanced by means of the Kinect sensor. Taking account of these topics, we can say that Kinect shows a great potential for academic studies in many areas

\section{Kinect Skeletal Tracking}

The key to the innovation behind Kinect is its advanced skeletal tracking ability. In skeletal tracking, RGB and depth streams are processed by the software to create a visual stick skeleton to represent the human body. Skeleton is constructed by a number of joints representing body parts such as head, neck, shoulders, and arms. After calculating 3D coordinates of all joints, Kinect succeeds at determining all the 3D parameters of these joints in real time to allow fluent interactivity [5]. Fig. 3 shows all the joints and their labels supported by Kinect for Xbox One sensor and the constructed skeleton map [7].

\section{LITRATURE SURVEY}

Shoulder injuries are very regular in sports and certain labour concentrated occupations. While some injuries are minor and full recovery is within 1-2 weeks, some major injuries requires the person to consult a physiotherapist and follow an exercise plan. Hidden Markov Models (HMM) for recognition and a histogram-based comparison for computing the accuracy score. The Microsoft Kinect sensor is used to obtain 3D coordinates of human joints. Important features are extracted from the skeletal coordinates which are then quantized into 16 intermediate upperbody poses. It intends to help the patient by keeping track of daily exercise routine, advising for improvements and maintaining records for doctor to access. [1] The difficulty of vision-based posture estimation is really decreased with the aid of profitable depth camera, such as Microsoft Kinect. Yet, there is still much to do to bridge the results of human posture estimation and the understanding of human movements. The experiments are conducted on the videos of ten action types, and the results show that the proposed human action descriptor is representative for action video retrieval and the tutor 
system can successfully help the user while learning action movements [2] The design and implementation of a Kinect-based system for rehabilitation exercises monitoring and guidance has been developed in this paper. The Unity framework frame work has been used to implement system because it enables to use virtual reality techniques to demonstrate detailed movements to the patient, and to facilitate examination of the quality and quantity of the patient sessions by the clinician. A set of basic rule elements has been developed that can be used to express the correctness rules for common rehabilitation exercises [3].

\section{SYSTEM ARCHITECTURE}

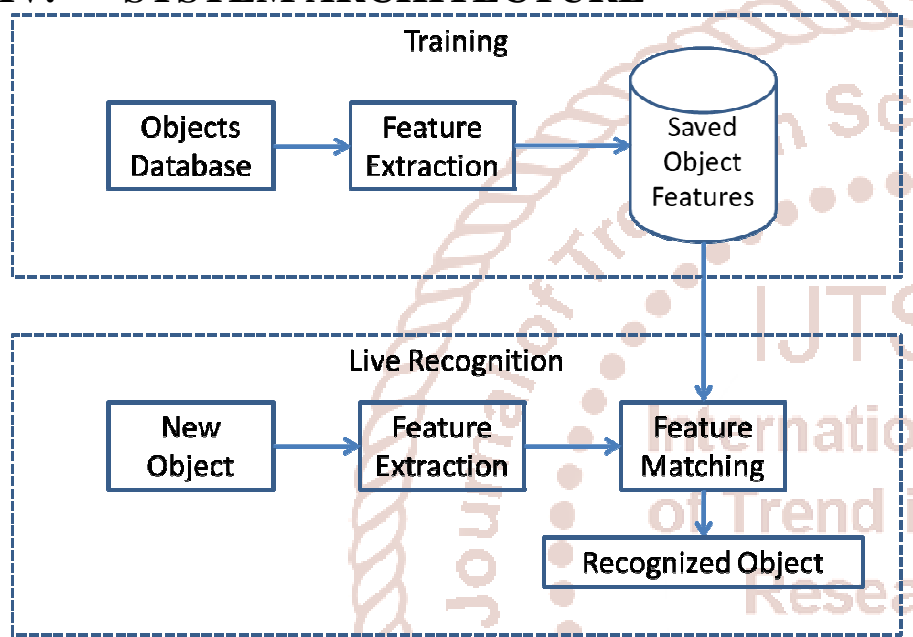

Fig.2. Kinet based Physical Rehabilitation system

Kinet based automatic physical rehabilitation system is shown in fig.2, The system consisting of two phase. Training phase and Live Recognition phase. The proposed algorithm requires a set of data where it trains the exercise model. The data is trained by machine learning .During recognition phase the image is captured and then skeleton is detected then the data is compared and classified by using support vector machine.

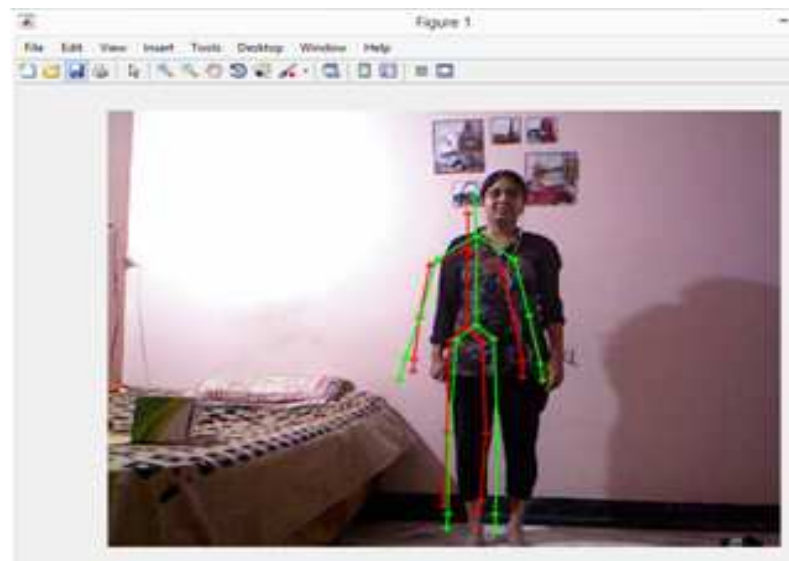

Fig .3 Exercise pose 1

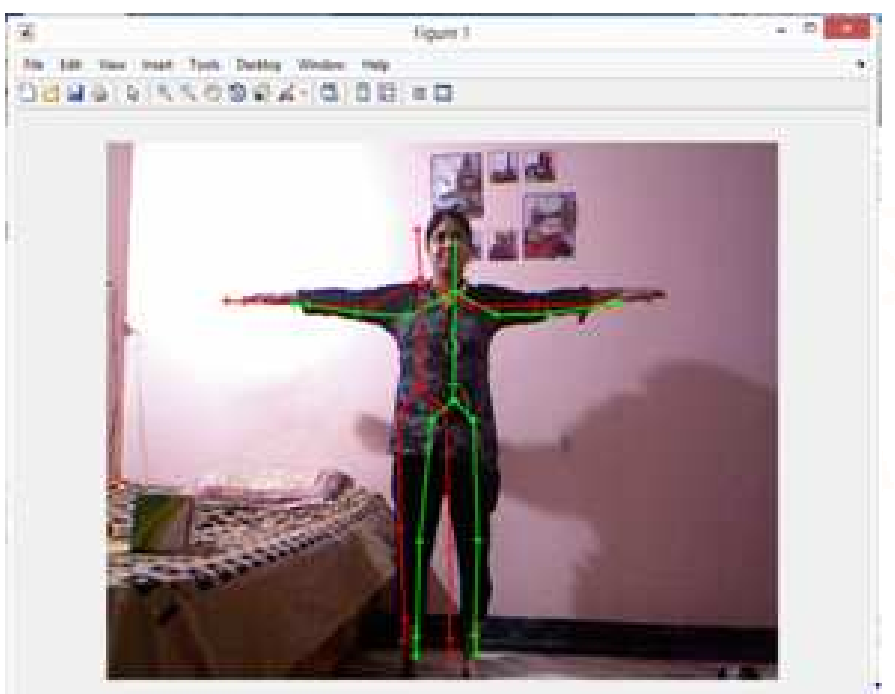

Fig .4 Exercise pose 2

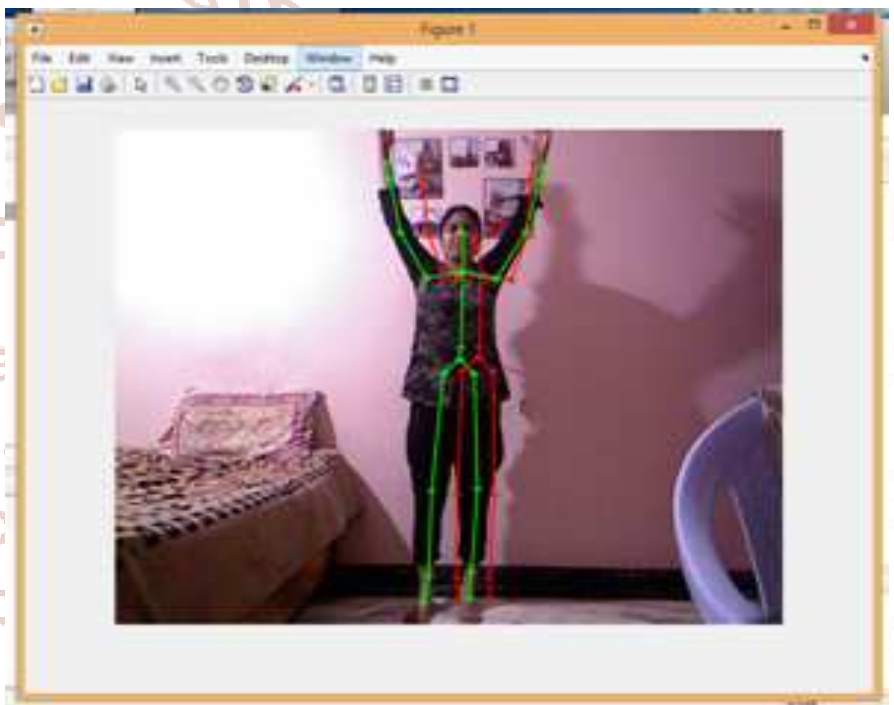

Fig.5 Exercise pose 3.

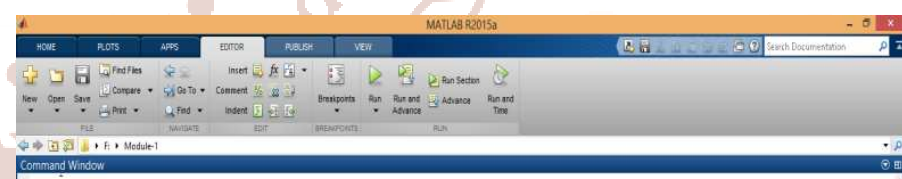

द 9 का $\Theta 4$

Fig.5. Command window showing completion of Exercise.

A Kinect-based rehabilitation system utilized to record their movements. These movements are captured by color image, depth image and skeleton. 
The image is tracked. performed by the coaches are considered as standard movement, and the skeleton of these movements are used as a feature and trained by Support vector machine. During recognition process the new feature are extracted and compared with the database trained by SVM. The classification is done by multisvm. When a person uses this rehabilitation system, the system first displays standard movements on the screen. Then person follows the information on screen to perform compared to the movements of coaches by comparing the skeletons. The results will show on screen to inform the user regarding exercise is completed. The message is shown in Figure 5.

Result:
\begin{tabular}{|c|c|c|c|c|}
\hline $\begin{array}{c}\text { Sr. } \\
\text { No }\end{array}$ & Exercise & $\begin{array}{r}\text { No. Of } \\
\text { Attempts }\end{array}$ & $\begin{array}{c}\text { Coorect / } \\
\text { Incorrect } \\
\text { Detection }\end{array}$ & $\begin{array}{c}\text { Accuracy } \\
(\%)\end{array}$ \\
\hline 1 & Pos. 1 & 10 & 10 & 100 \\
\hline 2 & Pos. 2 & 10 & 10 & 100 \\
\hline 3 & Pos. 3 & 10 & 10 & 100 \\
\hline \multicolumn{7}{|c|}{ Total Accuracy } & \multicolumn{2}{|c|}{$100 \%$} \\
\hline
\end{tabular}

\section{CONCLUSION}

Thus, the paper proposes to develop a physical training system which allows users to perform exercises under computer supervision anywhere, thereby, reducing the need for users to travel to the gym or to the physiotherapist to perform basic routine exercises. This system promotes health and fitness amongst the youth and elderly alike. If used regularly, it serves as a guide and mentor to perform certain compound movements accurately, eliminating the possibility of injuries that may be caused due to incorrect form.

\section{REFERENCES:}

1. Development of a Kinect-Based Physical Rehabilitation System Param Uttarwar t, Deepak Mishra, t Space Applications Centre, Ahmedabad, India, Department of Avionics, Indian Institute of Space.

2. Real-Time Human Movement Retrieval and Assessment With Kinect Sensor Min-Chun $\mathrm{Hu}$, Member, IEEE, Chi-Wen Chen, Wen-Huang Cheng, Member, IEEE, Che-Han Chang, Jui-Hsin Lai, and Ja-Ling Wu, Fellow, IEEE

3. D. Tino and C. Hillis, "The full can exercise as the recommended exercise for strengthening the supraspinatus while minimizing impingement," Strength \& Conditioning Journal, vol. 32, no. 5, pp. 33-35, 2010.

4. A. Mobini, S. Behzadipour, and M. Saadat Foumani, "Accuracy of kinect's skeleton tracking for upper body rehabilitation applications," Disability and Rehabilitation: Assistive Technology, no. 0, pp. 1-9, 2013.

5. R. Lun and W. Zhao, "A survey of human body motion tracking based on kinect," International Journal of Pattern Recognition and Artificial Intelligence (submitted), 2014.

6. Rob Miles.Start Here! Learn the Kinect API, 1stEdition, 2012.

7. Untitled Photograph of Kinect Sensors on Human Body ,Retrieved March 10, 2013 from: http://gmv.cast.uark.edu/uncategorized/working with -data - from - the -kinect/attachment/kinectsensors -on-human-body/.]

8. Samet Erap. Gesture-Based PC Interface With Kinect Sensor, 2012.

9. D. McNeill, Language and gesture vol. 2: Cambridge Univ, Pr, 2000. 